\title{
Assessing patient preferences in heart failure using conjoint methodology
}

\author{
This article was published in the following Dove Press journal: \\ Patient Preference and Adherence \\ 26 August 2015 \\ Number of times this article has been viewed
}

\author{
Giovanni Pisa' \\ Florian Eichmann' \\ Stephan Hupfer ${ }^{2}$ \\ 'Kantar Health GmbH, Munich, \\ Germany; ${ }^{2}$ Novartis Pharma GmbH, \\ Nuernberg, Germany
}

\begin{abstract}
Aim: The course of heart failure (HF) is characterized by frequent hospitalizations, a high mortality rate, as well as a severely impaired health-related quality of life (HRQoL). To optimize disease management, understanding of patient preferences is crucial. We aimed to assess patient preferences using conjoint methodology and HRQoL in patients with HF.

Methods: Two modules were applied: an initial qualitative module, consisting of in-depth interviews with $12 \mathrm{HF}$ patients, and the main quantitative module in $300 \mathrm{HF}$ patients from across Germany. Patients were stratified according to the time of their last HF hospitalization. Each patient was presented with ten different scenarios during the conjoint exercise. Additionally, patients completed the generic HRQoL instrument, EuroQol health questionnaire (EQ-5D $\left.{ }^{\mathrm{TM}}\right)$.

Results: The attribute with the highest relative importance was dyspnea (44\%), followed by physical capacity (18\%). Of similar importance were exhaustion during mental activities (13\%), fear due to HF (13\%), and autonomy (12\%). The most affected HRQoL dimensions according to the EQ-5D questionnaire were anxiety/depression (23\% with severe problems), pain/discomfort (19\%), and usual activities (15\%). Overall average EQ-5D score was 0.39 with stable, chronic patients (never hospitalized) having a significantly better health state vs the rest of the cohort. Conclusion: This paper analyzed patient preference in HF using a conjoint methodology. The preference weights resulting from the conjoint analysis could be used in future to design HRQoL questionnaires which could better assess patient preferences in HF care.
\end{abstract}

Keywords: heart failure, quality of life, conjoint analysis, utility, patient preference

\section{Introduction}

Heart failure (HF) is among the most frequent and cost-intensive chronic diseases ${ }^{1}$ with $1 \%-2 \%$ of the population in developed countries affected and prevalence rising to $>10 \%$ in the age group $\geq 70$ years. The course of the disease is characterized by frequent hospitalizations and high mortality rate, reaching $40 \%$ in the 5 years following a hospitalization for HF. ${ }^{2,3}$ In addition, HF patients suffer from a poor health-related quality of life (HRQoL) that is comparable to advanced cancer patients. ${ }^{4} \mathrm{HRQoL}$ is affected by different factors, including dyspnea, physical limitations, depression, and fear. However, the relative importance of these factors is currently unclear. Thus, to optimize disease management strategies and to support development of interventions meeting patients' needs, understanding of patient preferences is crucial.

Conjoint analysis, particularly choice-based and discrete choice designs, can be used to quantify preferences for several aspects of an intervention. Conjoint analysis methods are particularly useful for quantifying preferences for nonmarket goods and services or where market choices are severely constrained by regulatory and institutional factors, such as in health care. ${ }^{5}$ Conjoint analysis surveys are increasingly used in the study of efficacy-safety tradeoffs, for example, in drug-development decisions.
Correspondence: Giovanni Pisa Kantar Health $\mathrm{GmbH}$, Landsberger Strasse 284, 80687 Munich, Germany Tel +498956001958

$\mathrm{Fax}+49895600 \quad 1400$

Email giovanni.pisa@kantarhealth.com 
They offer an empirical solution to estimating a person's willingness to accept such tradeoffs. Choice-based experiments to identify patient preferences have been established for several years. ${ }^{6,7}$ Here, patients choose their preferred options within the so-called scenarios, reflecting several "attributes" that are important for the selection of a certain scenario. According to recent results from other indications (eg, chronic obstructive pulmonary disease), choice-based experiments have proven to be useful to gain understanding of disease and treatment-related factors that are important to define patient populations.

While a US-based study analyzed preferences for treatment outcomes in HF patients, in terms of tradeoff of symptoms vs survival, ${ }^{8}$ no study so far to our knowledge has examined detailed preferences of HF patients concerning the disease attributes which jointly determine their quality of life. We therefore designed a study to assess the relative importance of HF symptoms and accompanying features from the patients' perspective. We used the choice-based experiment as an indirect approach, since revealing preferences through choices has proven to be more suitable for assessing preferences than employing a direct approach.

In line with recent work, preference weights resulting from the conjoint analysis can be used to develop a patientreported outcome instrument that can be used in patient-based studies such as clinical trials or registries. ${ }^{9}$

\section{Methods}

\section{Study design}

This preference-based study analyzed subjects' opinion regarding disease and treatment in an anonymized way and in accordance with the applicable standard for opinion research: ISO 20252. After a preliminary qualitative research module, a quantitative data assessment was conducted. To ensure consistency and (high) quality of interviews, interviewers were trained by the core project team via a central telephone briefing. During this briefing, all details regarding the interviewing process were explained in detail, including the main interview tools used. The study was conducted in Germany. All patients provided written informed consent to participate.

\section{Qualitative module}

Twelve HF patients participated in the preliminary qualitative assessment. The objective was to gain insights into patients' health state (attitudes toward HF, symptom profiles, unmet needs, and preferences for HF drugs to be developed) to be used for the generation of attributes within the conjoint exercise in the main research module.
All participants were recruited through their treating physicians. The main inclusion criteria for this survey were disease severity (New York Heart Association [NYHA] class II-IV), age (four patients were 60-69 years of age, eight patients were $\geq 70$ years of age), and a history of hospitalization for HF. Four patients who had been hospitalized for HF within 3 months of their study recruitment, four patients who had been admitted for HF at least once ( $>3$ months), and four stable chronic patients (never hospitalized) were interviewed. The interviews were conducted at home, face-to-face, with an average duration of 45 minutes. The results of these qualitative interviews were highly consistent and in line with a priori expectations. They served as a framework for the design of the conjoint exercise for the quantitative phase.

\section{Quantitative module}

In the quantitative research module, 300 patients with HF (NYHA stage II-IV) from across Germany participated: $\mathrm{n}=100$ patients having experienced a hospitalization within the last 3 months, $n=100$ patients having experienced at least one hospitalization ( $>3$ months), and $n=100$ stable chronic patients (never hospitalized), to ensure an equal distribution of these subgroups within the overall sample. The sample was also equally split into age groups, with each third of patients being $60-69,70-75$, and over 75 . As in the qualitative module, we conducted face-to-face interviews in patient's home. The average total length of the interviews was 20 minutes, of which approximately 15 minutes was dedicated to the conjoint exercise. The patients were completely independent in their answering behavior, since the interviewer only explained the conjoint exercise. No drop-outs were observed during the interviewing process.

In the choice-based conjoint (CBC) exercise, the following attributes (and their levels) were considered:

1. Dyspnea

2. Physical capacity in daily activities

3. Exhaustion during mental activities

4. Autonomy

5. Fear due to HF

These attributes and their respective levels were determined in line with current understanding of HF symptomatology $y^{1,2}$ and through the previously mentioned qualitative research module with HF patients. The list of attributes and attribute levels used in the conjoint is shown in Table 1.

The current range of attribute levels was chosen to ensure that all levels were evenly graded and spaced. Before starting the main phase, five pilot interviews were conducted faceto-face to check with patients the clarity and comprehension 
Table I Attributes and levels of the conjoint design

\begin{tabular}{ll}
\hline Attributes & Levels \\
\hline Dyspnea & Never dyspnea, except on increased physical exertion \\
\hline & Dyspnea during exertion \\
3 & Dyspnea at walking pace \\
4 & Dyspnea during slightest physical exertion \\
5 & Dyspnea at rest \\
Exhaustion during mental activities* \\
I \\
2 & No exhaustion \\
3 & Mild exhaustion \\
4 & Strong exhaustion \\
Physical capacity in daily activities \\
I \\
2 & Can freely organize my day \\
3 & Can freely organize most of my day \\
4 & Can organize my day only partly \\
Autonomy & Cannot organize my day at all \\
I & No assistance necessary \\
2 & Sometimes assistance necessary \\
3 & Frequent assistance necessary \\
4 & Rely on permanent assistance \\
Fear due to heart failure \\
I & No fear \\
2 & Have fear sometimes \\
3 & Have fear often \\
4 & Permanent fear/panic \\
\hline Note: &
\end{tabular}

Note: *For example, active participation in discussions, reading, using computer and watching TV.

of the choice tasks. Also, the interview length was tracked to ensure that the cognitive load of patients is well balanced: from previous $\mathrm{CBC}$ studies, we know that a length of 15 minutes permits a reliable answering behavior without overloading the respondent. All scenarios shown to respondents were computer-generated: the number of scenarios to be shown was generated following the guidelines of the Sawtooth Software Technical Paper. ${ }^{10}$ In this case, sample size, number of attributes, and attribute levels were defined in such a way that ten scenarios resulted from the calculation.

Furthermore, ten different computer-generated versions of the conjoint exercise (each containing these ten scenarios) were used to ensure that the study design is well balanced across all respondents. The experimental design was balanced across all design versions. The different versions are not simply a randomization of the different choice tasks, but each version contained a different set of choice tasks to ensure that all attribute levels are balanced across all versions. The ten versions of the conjoint exercise were uniformly distributed across the respondents (each version for $\mathrm{n}=30$ patients).

Each patient was presented with ten different scenarios (choice tasks) during the conjoint exercise. A choice task consisted of three patient profiles (defined by the systematic variation of the attribute levels) among which the patient had to choose (Figure 1).

The patients were asked: "In the following I will show you 10 sheets. Each sheet describes the current situation of three persons A, B, and C. Their present situation is primarily influenced by the current treatment of their HF. Please select the one patient being in the best health state according to your opinion".

Additional to the conjoint exercise, patients completed the generic HRQoL instrument, EuroQol health questionnaire $\left(\mathrm{EQ}-5 \mathrm{D}^{\mathrm{TM}}\right)$. EQ-5D is a standardized instrument for use as a measure of health outcome and to derive health state utilities. EuroQol health questionnaire (EQ-5D ${ }^{\mathrm{TM}}$ ) was developed by the EuroQoL group as a generic instrument for describing and investigating HRQoL. ${ }^{11}$ The EQ-5D is applicable to a wide range of health conditions and treatments, and it provides a simple descriptive profile and a single index value for health status. It is based on a descriptive system that defines health in terms of five dimensions: mobility, self-care, usual activities,

\begin{tabular}{|c|c|c|c|}
\hline \multicolumn{4}{|l|}{ Version 1, Task 1} \\
\hline \multicolumn{4}{|c|}{ Please select the one person being in the best situation according to your opinion } \\
\hline Patient & A & B & C \\
\hline Dyspnea & $\begin{array}{c}\text { Dyspnea at walking } \\
\text { pace }\end{array}$ & $\begin{array}{c}\text { Dyspnea during } \\
\text { slightest physical exertion }\end{array}$ & $\begin{array}{l}\text { Never dyspnea, except on } \\
\text { increased physical exertion }\end{array}$ \\
\hline $\begin{array}{l}\text { Physical capacity in } \\
\text { daily activities }\end{array}$ & $\begin{array}{c}\text { Cannot organize my } \\
\text { day at all }\end{array}$ & $\begin{array}{c}\text { Can freely organize } \\
\text { most of my day }\end{array}$ & $\begin{array}{c}\text { Can organize my day } \\
\text { only partly }\end{array}$ \\
\hline $\begin{array}{l}\text { Exhaustion during } \\
\text { mental activities }\end{array}$ & Strong exhaustion & Mild exhaustion & No exhaustion \\
\hline Autonomy & $\begin{array}{c}\text { Frequent assistance } \\
\text { necessary }\end{array}$ & $\begin{array}{c}\text { Rely on permanent } \\
\text { assistance }\end{array}$ & $\begin{array}{c}\text { Sometimes assistance } \\
\text { necessary }\end{array}$ \\
\hline \multirow[t]{2}{*}{ Fear due to HF } & Have fear sometimes & Permanent fear/panic & Have fear often \\
\hline & O & $\bigcirc$ & $\mathrm{O}$ \\
\hline
\end{tabular}

Figure I Choice task example. Abbreviation: HF, heart failure. 
pain/discomfort, and anxiety/depression. ${ }^{12}$ The EQ-5D questionnaire was completed by respondents, and the corresponding index was calculated, indicating the patient's health status.

\section{Study measures - quantitative module Sociodemographics}

Each respondent provided information with respect to his/her sex, age, education, employment status, and federal state.

\section{Health status}

The EQ-5D questionnaire was included as a measure of health status. The utility values and the visual analog scale (VAS) scores, derived from the EQ-5D items, were calculated.

\section{Patient preferences}

The relative importance of HF attributes and the utilities for the attribute levels (part-worth utilities) were obtained from the conjoint analysis.

\section{Statistical analysis}

- The EQ-5D utilities and VAS scores were calculated according to the guidelines of EuroQoL group. ${ }^{12}$

- In the current setting, parameter estimates are based on a $\mathrm{CBC}$ analysis. $\mathrm{CBC}$ is a full-profile conjoint model where attribute (levels) independencies are assumed in the model structure. Estimates were derived by a hierarchic Bayes regression (Supplementary material) based on a mixed multinomial logit model. ${ }^{13}$ Considerations on sample size were based on measures of convergence of estimations: an overall sample size of $n=300$ was required to allow preferences to be estimated with a precision of $\pm 2 \%$.

- Differences between patient subgroups were analyzed by two-tailed $t$-tests on column means and proportions.

\section{Results \\ Qualitative module}

According to the qualitative interviews with patients, the most distressing aspects of HF are shortness of breath, exhaustion, and the corresponding limitations in conducting daily activities (eg, going to work, home activities, sometimes even going for a walk). The mental situation of HF patients is dominated by the anxiety of experiencing an episode of shortness of breath: the absence of such episodes and hence having a more active life and feeling more fit are what patients desire most to improve their quality of life.

\section{Quantitative module}

The most relevant patient characteristics are represented in Table 2 .
The cohort studied was predominantly male and elderly with two-third of the patients being 70 years of age or older by study design. It is noteworthy that in patients having experienced a hospitalization within the last 3 months, there was a smaller proportion of patients older than 75 years than in the other two cohorts (19\% vs $42 \%$ and $39 \%)$.

Noteworthy are also the differences regarding the NYHA class among the subgroups: while NYHA class II was predominant in chronic patients $(77 \%$ vs $51 \%$ and $30 \%$ for the patient categories "never hospitalized" vs "hospitalized $>3$ months" and "hospitalized $\leq 3$ months", respectively), NYHA class III and IV were mainly present in patients with a hospitalization $\leq 3$ months $(64 \%, 48 \%$, and $23 \%$ for class III and $6 \%$, $1 \%$, and $0 \%$ for class IV for patients "hospitalized $\leq 3$ months", "hospitalized $>3$ months", and "never hospitalized", respectively). Patients with a more distant hospitalization ( $>3$ months) presented with an intermediate NYHA class phenotype.

Otherwise, no significant differences were noted between the three patient subgroups.

\section{Health status}

The average EQ-5D index was 0.39 , and the average VAS score was 49.5 for the total patient sample, indicating a generally poor HRQoL among participating HF patients.

Stable chronic patients (never hospitalized) had a significantly better health state assessed by EQ-5D index (0.51), compared to the total sample.

On the other hand, patients having experienced a hospitalization within the last 3 months had a significantly worse health state than the total sample (0.31).

Detailed results of the EQ-5D utilities and VAS scores are included in Table 3.

\section{$\mathrm{CBC}$ analysis and patient preferences}

Three hundred patients took part in the conjoint analysis. According to the participants, the attribute with the highest relative importance was (the absence of) dyspnea (44\%), followed by physical capacity in daily activities (18\%).

Symptoms reported as being of similar importance were exhaustion during mental activities (eg, active participation in discussions, reading, using a computer, watching TV) (13\%), fear due to HF (13\%), and autonomy (12\%).

An overview of the relative importance of the attributes is included in Table 4.

The part-worth utility values are important indicators of the effect of the attribute levels on health state preference for HF.

To never have dyspnea (or at least only during exertion) would be very preferable for the patients. Dyspnea at rest had a very negative impact on patient preferences. 
Table 2 Patient's baseline characteristics

\begin{tabular}{|c|c|c|c|c|}
\hline Variable & $\begin{array}{l}\text { Total } \\
\text { patients, } \\
\mathrm{N}=\mathbf{3 0 0} \\
(\%)\end{array}$ & $\begin{array}{l}\text { Patients having } \\
\text { experienced a } \\
\text { hospitalization } \\
\text { within the last } \\
3 \text { months, } n=100(\%)\end{array}$ & $\begin{array}{l}\text { Patients having } \\
\text { experienced at least one } \\
\text { hospitalization } \\
\text { (>3 months), } n=100(\%)\end{array}$ & $\begin{array}{l}\text { Stable chronic patients } \\
\text { (never hospitalized), } \\
n=100(\%)\end{array}$ \\
\hline \multicolumn{5}{|l|}{ Sex } \\
\hline Male & 74 & 75 & 73 & 75 \\
\hline Female & 26 & 25 & 27 & 25 \\
\hline \multicolumn{5}{|l|}{ Age (years) } \\
\hline 60-69 & 33 & 31 & 38 & 31 \\
\hline 70-75 & 33 & $50 *$ & $23^{*}$ & 27 \\
\hline Over 75 & 33 & $19 *$ & 39 & $42^{*}$ \\
\hline \multicolumn{5}{|l|}{ NYHA class } \\
\hline II & 53 & $30 *$ & 51 & $77^{*}$ \\
\hline III & 45 & $64^{*}$ & 48 & $23^{*}$ \\
\hline IV & 2 & $6^{*}$ & 1 & 0 \\
\hline \multicolumn{5}{|l|}{ School education (highest level) } \\
\hline $\begin{array}{l}\text { Higher education entrance qualification } \\
\text { not further specified ("Hochschulreife") }\end{array}$ & 8 & 5 & 9 & 9 \\
\hline $\begin{array}{l}\text { Technical college entrance qualification } \\
\text { ("Fachhochschulreife") }\end{array}$ & 7 & 7 & 6 & 8 \\
\hline $\begin{array}{l}\text { University entrance qualification } \\
\text { (“Abitur”) }\end{array}$ & 17 & 13 & 19 & 20 \\
\hline $\begin{array}{l}\text { General certificate of secondary } \\
\text { education ("Realschulabschluss") }\end{array}$ & 28 & 35 & 26 & 24 \\
\hline $\begin{array}{l}\text { Certificate of basic secondary } \\
\text { education ("Hauptschulabschluss") }\end{array}$ & 40 & 40 & 40 & 39 \\
\hline \multicolumn{5}{|l|}{ Employment status } \\
\hline Employed & 6 & $I^{*}$ & $11^{*}$ & 6 \\
\hline Unemployed & 5 & 9 & 3 & 4 \\
\hline Retired & 89 & 90 & 86 & 90 \\
\hline \multicolumn{5}{|l|}{ Regional distribution } \\
\hline Northern Germany & 16 & 12 & 18 & 19 \\
\hline Southern Germany & 23 & 23 & 25 & 21 \\
\hline Western Germany & 40 & 39 & 39 & $4 I$ \\
\hline Eastern Germany & 21 & 26 & 18 & 19 \\
\hline
\end{tabular}

Note: $* P<0.05$ (subgroup vs total sample).

Abbreviation: NYHA, New York Heart Association.

Table 3 EQ-5D ${ }^{\mathrm{TM}}$ utilities and VAS scores: differences between post-acute and chronic patients (with and without hospitalization)

\begin{tabular}{lll}
\hline Total sample and subgroups & EQ-5D index & VAS score \\
\hline Total patients $(\mathrm{n}=300)$ & 0.39 & 49.5 \\
Patients having experienced & 0.31 & 43.7 \\
a hospitalization within & & \\
the last 3 months $(\mathrm{n}=100)$ & & $<0.05$ \\
$\quad P$-value & $<0.05$ & 52.0 \\
Patients having experienced & 0.36 & \\
at least one hospitalization & & \\
$\begin{array}{l}\text { ( }>3 \text { months) }(\mathrm{n}=100) \\
P \text {-value }\end{array}$ & $\mathrm{ns}$ & $\mathrm{ns}$ \\
$\begin{array}{l}\text { Stable chronic patients } \\
\text { (never hospitalized) }(\mathrm{n}=100)\end{array}$ & $0.5 \mathrm{I}$ & 52.9 \\
$P$-value & & \\
\hline
\end{tabular}

Note: $P$-values calculated vs total sample.

Abbreviations: EQ-5D, EuroQol health questionnaire; VAS, visual analog scale; ns, non significant.
To be able to freely organize their day (or most of it) represented a big benefit for the patients. Apparently, a limited autonomy and a light exhaustion during mental activities could be accepted by the patients. The idea of living with a permanent fear/panic due to HF had a very negative influence on patient preferences.

Detailed results are included in Figure 2.

\section{Discussion}

HF remains one of the major health challenges worldwide. While a focus in recent years has been to reduce mortality and morbidity of these patients, HRQoL is also poor and comparable to advanced cancer patients. ${ }^{4}$ While some questionnaires, for instance EQ-5D, ${ }^{11}$ Kansas City Cardiomyopathy 
Table 4 Relative importance of HF attributes (obtained from the conjoint analysis)

\begin{tabular}{ll}
\hline Attribute & $\begin{array}{l}\text { Relative } \\
\text { importance (\%) }\end{array}$ \\
\hline Dyspnea & 44 \\
Physical capacity in daily activities & 18 \\
Exhaustion during mental activities & 13 \\
Fear due to HF & 13 \\
Autonomy & 12 \\
\hline
\end{tabular}

Note: Total number of patients, $\mathrm{N}=300$.

Abbreviation: $\mathrm{HF}$, heart failure.

Questionnaire, ${ }^{14}$ or Minnesota Living with Heart Failure Questionnaire, ${ }^{15}$ are available, it is unclear as to what extent they adequately mirror the aspects in HRQoL that are most important to patients. Choice-based experiments to identify patient preferences ${ }^{6,7}$ can offer a way to better understand the main drivers of patient suffering and thus the main points that should be improved from a HRQoL point of view by therapeutic interventions.

Our conjoint study of patient preferences included a sample of 300 patients (quantitative phase) from all over Germany. The elderly, predominantly male population matches well with data obtained from the real-word European HF population as observed in the ESC-HF pilot study ${ }^{16}$ or the ESC Heart Failure Long-Term Registry. ${ }^{17}$

As expected, patients reported a generally poor HRQoL by both EQ-5D index and VAS score. Interestingly, HRQoL differed with respect to the patient's history of HF hospitalization. While patients with a recent hospitalization in the past 3 months reported the worst HRQoL, patients who had never experienced a hospitalization in the past seemed to do better both in terms of EQ-5D index and VAS, although absolute values were still poor. Although these results may be confounded to a certain degree by the reduced proportion of very elderly patients ( $>75$ years) among those with recent hospitalization, it does not seem implausible that the events and clinical profile associated with a recent hospitalization could lead to reduced HRQoL. Nevertheless, the mean EQ-5D index and VAS scores obtained in our study seem to be in line with the indexes and scores found in existing literature for cardiovascular disease: mean EQ-5D indexbased scores ranged from 0.24 to 0.90 , while VAS scores ranged from 37 to $89 .{ }^{18}$

The patient preferences resulting from the conjoint analysis show that patients desire most the absence of dyspnea and the physical ability to conduct daily activities.

Interestingly, dyspnea and performance capability seem to have high importance in other diseases as well: in a choice-based experiment conducted with chronic obstructive pulmonary disease patients, the relief of dyspnea was the most important attribute ( $36 \%$ vs $44 \%$ in our current study), followed by performance capability (19\% vs $18 \%$ for physical capacity in our current study). ${ }^{3}$

Furthermore, patients seem to cope with their limited autonomy, a certain level of fear due to their condition, and some degree of exhaustion during daily mental activities.

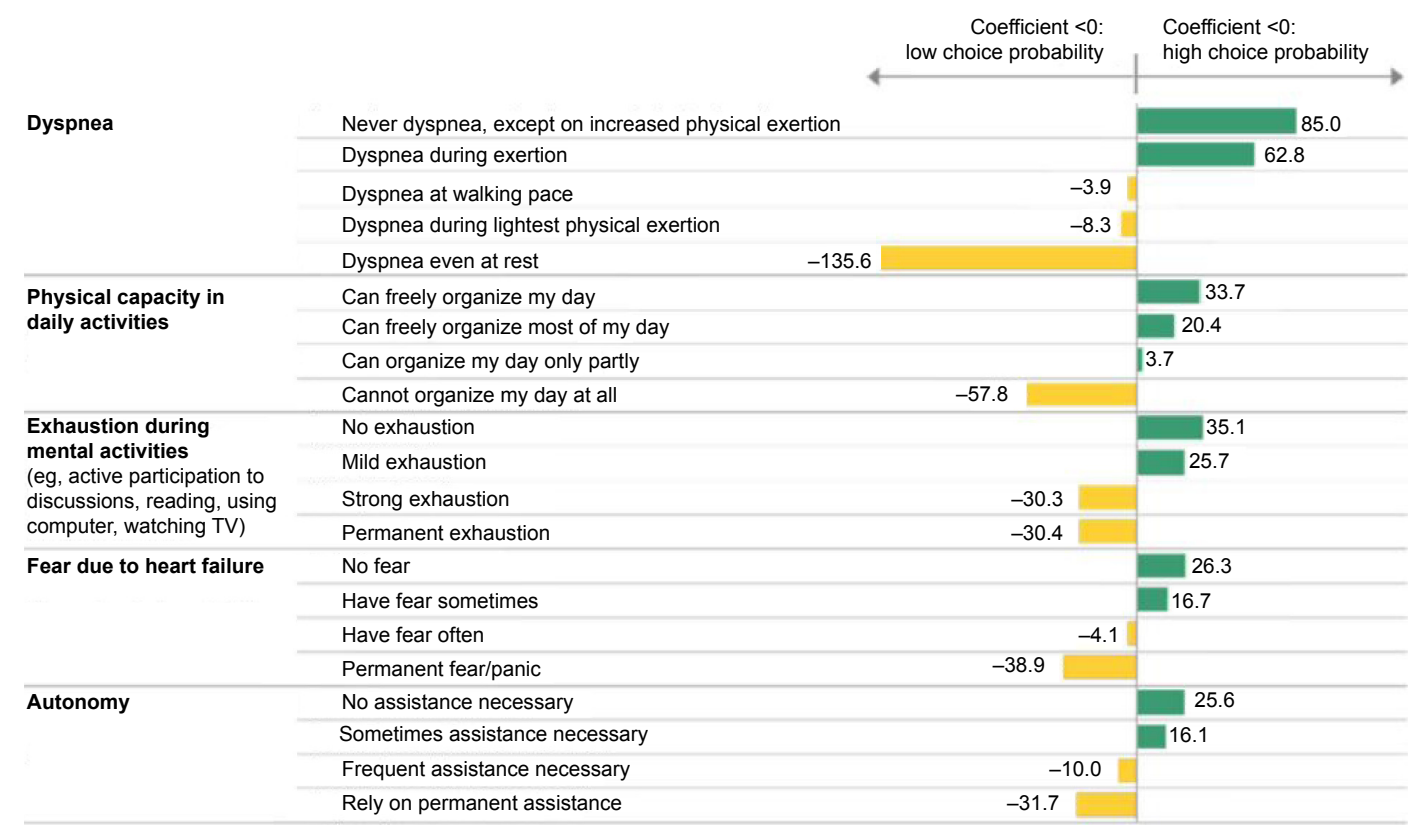

Figure 2 Effect of attribute levels on health state preference: part-worth utilities. 
Noteworthy is the high proportion of patients in the sample with anxiety and depression ( $23 \%$ with severe problems) according to the EQ-5D questionnaire: further, the part-worth utilities resulting from the conjoint analysis indicate that the idea of living with a permanent fear/panic due to HF has a very negative influence on patient preferences. This underlines the high psychological burden of HF on patients who already cope with a significant level of anxiety and are afraid of an increased anxiety due to their disease (Figure 2).

In the following part, we discuss the limitations and strengths of our study. Although due to feasibility reasons the time window for a population early after an acute episode was chosen at 3 months, a closer temporal connection to the acute episode might have led to greater differences in the chronic cohorts. A further limitation might be represented by the fact that many clinical characteristics (including reduced/preserved ejection fraction) as well as the time since diagnosis or comorbidities were not captured.

A strength of our study can be seen in the good age stratification in the sample. As mentioned above, the elderly and predominantly male population matches well with data obtained from the real-word European HF population.

Further, we used an established methodology to measure patient preferences for HF as opposed to generally ask patient for preferred aspects of their life with the disease: based on a choice-based experiment, several concrete health state scenarios were systematically presented to the patients who were able to make a free choice for their preferred health state.

Lastly, combining the use of a HRQoL instrument and of a choice-based experiment, the study could deliver important insights regarding both HF patients' life with their disease and their preference for several aspects of the disease and its symptomatology.

\section{Conclusion}

We identified attributes of HF being most relevant from the patients' point of view: the absence of dyspnea and the physical ability to conduct daily activities, which outperform other attributes - such as the fear due to their condition, the exhaustion during daily mental activities, and their limited autonomy - in a trade-off situation a patient might be faced with.

Particularly, dyspnea seems to outperform the other attributes in terms of importance. To never have dyspnea (or at least only during exertion) would be very preferable for the patients, and dyspnea at rest has a very negative impact on patient preferences. Since relief from dyspnea seems to play a key role in improving HF patient's HRQoL, it might be worthy to further explore this aspect in future studies and increase awareness concerning this important fact among health care providers. In particular, dyspnea per se may well be a valuable endpoint for trials in the chronic setting, similar to its use in acute HF trials.

Furthermore, we assessed that HF is associated with poor HRQoL, particularly for those patients with post-acute HF.

Given the substantial burden of HF, greater awareness of the condition and earlier intervention could help improve the quality of life of HF patients. The results of the conjoint analysis, specifically the preference weights, will be used to develop a patient-reported outcomes instrument to be used in future studies of HF.

\section{Acknowledgments}

The authors advise that this study did not require any ethical approval as it was a market research study. Novartis Pharma $\mathrm{GmbH}$ funded this study and provided financial support for medical editorial assistance. The abstract of this paper was presented at the Heart Failure Congress 2014 in Athens, Greece, during May 17-18, 2014, as a poster presentation with interim findings. The actual paper, however, has never been published.

\section{Author contributions}

GP organized the data collection. GP, FE, and SH designed the study. GP and FE performed the statistical analyses. All three authors contributed to the interpretation of the data, the preparation of the manuscript, and the critical revisions of the manuscript. Guarantor of the overall content is GP.

\section{Disclosure}

GP has received financial support from Novartis Pharma $\mathrm{GmbH}$ for study conception, travel to meetings for this study, as well as the preparation and review of the manuscript. GP and FE (who are employed by Kantar Health GmbH), conducted the study, the analysis, and the manuscript preparation on behalf of Novartis Pharma GmbH. SH is a full-time employee of Novartis Pharma GmbH.

\section{References}

1. McMurray JJ, Adamopoulos S, Anker SD, et al. ESC guidelines for the diagnosis and treatment of acute and chronic heart failure 2012: The Task Force for the Diagnosis and Treatment of Acute and Chronic Heart Failure 2012 of the European Society of Cardiology. Developed in collaboration with the Heart Failure Association (HFA) of the ESC. Eur J Heart Fail. 2012;14(8):803-869.

2. Dietz R. Herzinsuffizienz. Harrison: Innere Medizin. Manfred Dietel, Norbert Suttorp, Martin Zeitz, editors. Berlin: ABW-Wissenschaftsverlag; 2008.

3. Loehr LR, Rosamond WD, Chang PP, Folsom AR, Chambless LE. Heart failure incidence and survival (from the Atherosclerosis Risk in Communities study). Am J Cardiol. 2008;101:1016-1022. 
4. Bekelman DB, Rumsfeld JS, Havranek EP, et al. Symptom burden, depression, and spiritual well-being: a comparison of heart failure and advanced cancer patients. J Gen Intern Med. 2009;24:592-598.

5. Torrance GW. Measurement of health state utilities for economic appraisal. J Health Econ. 1986;5(1):1-30.

6. Ryan M. Discrete choice experiments in health care. BMJ. 2004; 14(328):360-361.

7. Ryan M, Farrar S. Using conjoint analysis to elicit preferences for health care. BMJ. 2000;320(7248):1530-1533.

8. Stanek EJ, Oates MB, McGhan WF, Denofrio D, Loh E. Preferences for treatment outcomes in patients with heart failure: Symptoms versus survival. J Card Fail. 2000;6(3):225-232.

9. Pisa G, Freytag S, Schandry R. Chronic obstructive pulmonary disease (COPD) patients' disease-related preferences - a study using conjoint analysis. Patient. 2013;6(2):93-101.

10. Kuhfeld WF. Marketing Research Methods in SAS. Experimental Design, Choice, Conjoint, and Graphical Techniques. SAS 9.2 ed. Cary, NC: SAS Institute Inc.; 2010.

11. Herdman M, Gudex C, Lloyd A, et al. Development and preliminary testing of the new five-level version of EQ-5D (EQ-5D-5L). Qual Life Res. 2011;20(10):1727-1736.

12. Brooks R. EuroQoL: the current state of play. Health Policy. 1996; 37(1):53-72.
13. McFadden D, Train K. Mixed MNL models for discrete response. J Appl Econ. 2000;15(5):447-470.

14. Green CP, Porter CB, Bresnahan DR, Spertus JA. Development and evaluation of the Kansas City Cardiomyopathy Questionnaire: a new health status measure for heart failure. J Am Coll Cardiol. 2000;35: 1245-1255.

15. Rector TS, Kubo SH, Cohn JN. Patients self-assessment of their congestive heart failure: content, reliability and validity of a new measure, the Minnesota Living with Heart Failure questionnaire. Heart Fail. 1987;3:198-209.

16. Maggioni A, Dahlström U, Filippatos G. EURObservational Research Programme: the Heart Failure Pilot Survey (ESC-HF pilot). Eur J Heart Fail. 2010;12:1076-1084.

17. Maggioni AP, Anker SD, Dahlström U, et al; Heart Failure Association of the ESC. Are hospitalized or ambulatory patients with heart failure treated in accordance with European Society of Cardiology guidelines? Evidence from 12440 patients of the ESC Heart Failure Long-Term Registry. Eur J Heart Fail. 2013;15:1173-1184.

18. Dyer MT, Goldsmith KA, Sharples LS, Buxton MJ. A review of health utilities using the EQ-5D in studies of cardiovascular disease. Health Qual Life Outcomes. 2010;8:13. 


\section{Supplementary materials Hierarchic Bayes regression}

To make this model more explicit, we define some notation. We assume that individual part worths have the multivariate normal distribution,

$$
\beta_{i} \sim \operatorname{Normal}(\alpha, D)
$$

where $\beta_{i}$ is a vector of part worths for the $i$ th individual, $\alpha$ is a vector of means of the distribution of individuals' part worths, and $D$ is a matrix of variances and covariances of the distribution of part worths across individuals.

At the individual level, choices are described by a multinomial logit model. The probability of the $i$ th individual choosing the $k$ th alternative in a particular task is:

$$
p_{k}=\exp \left(x_{k}^{\prime} \beta_{i}\right) / \Sigma j \exp \left(x_{j}^{\prime} \beta_{i}\right)
$$

where $p_{k}$ is the probability of an individual choosing the $k$ th concept in a particular choice task and $x_{j}$ is a vector of values describing the $j$ th alternative in that choice task.

In words, this equation says that to estimate the probability of the $i$ th person's choosing the $k$ th alternative (by the familiar process used in many conjoint simulators), we do the following:

1. Add up the part worths (elements of $\beta_{i}$ ) for the attribute levels describing the $k$ th alternative (more generally, multiply the part worths by a vector of descriptors of that alternative) to get the $i$ th individual's utility for the $k$ th alternative,

2. Exponentiate that alternative's utility,

3. Perform the same operations for other alternatives in that choice task, and

4. Percentage the result for the $k$ th alternative by the sum of similar values for all alternatives.

The parameters to be estimated are the vectors $\beta_{i}$ of part worths for each individual, the vector $\alpha$ of means of the distribution of worths, and the matrix $D$ of the variances and covariances of that distribution. Please refer to the Sawtooth Software Technical Paper series for more information. ${ }^{1}$

\section{Reference}

1. Sawtooth Software; technical paper series. The CBC/HB System for Hierarchical Bayes Estimation Version 5.0 Technical Paper. Sawtooth Software, Inc. Available from: http://www.sawtoothsoftware. com/support/technical-papers/hierarchical-bayes-estimation/cbchb-technical-paper-2009. Accessed May 7, 2015.
Patient Preference and Adherence

\section{Publish your work in this journal}

Patient Preference and Adherence is an international, peer-reviewed, open access journal that focuses on the growing importance of patient preference and adherence throughout the therapeutic continuum. Patient satisfaction, acceptability, quality of life, compliance, persistence and their role in developing new therapeutic modalities and compounds to optimize

\section{Dovepress}

clinical outcomes for existing disease states are major areas of interest for the journal. This journal has been accepted for indexing on PubMed Central. The manuscript management system is completely online and includes a very quick and fair peer-review system, which is all easy to use. Visit http://www. dovepress.com/testimonials.php to read real quotes from published authors. 